\title{
Cidade Dinâmica
}

\section{Ferramentas digitais em prol do planejamento urbano}

\author{
Keila Fernanda Gomes Caldeira \\ Universidade Federal de Ouro Preto, Brasil \\ caldeira.keila@gmail.com \\ Rafael Lemieszek Pinheiro \\ Universidade Federal de Ouro Preto, Brasil \\ lemieszek@gmail.com
}

\begin{abstract}
Considering the variety of digital tools available nowadays and the growth of their possible uses in architecture and urbanism, we propose to study their potential as auxiliary tools in the process of urban design, based on their ability to help understand and take part in complex projects that are currently out of reach, technically or literally, to the general population.

This article analyzes the origins and the theoretical framework behind urban planning in the Brazilian contemporary cities, currently based on Euclidean Zoning, and the processes of connecting contributions from participatory process and digital tools into city-planning level decisions. attempting to have a closer look into the questions behind a new proposal of planning, on a non-euclidean way, where the urban parameters could be evaluated and defined individually for each plot, based on a set of parameters that vary gradually and are dynamic in nature.
\end{abstract}

Keywords: Euclidean planning; Participatory proccess; Digital tools; Parametric urban planning; Computational urbanism

\section{Introdução}

Este artigo tem como objetivo explorar o uso das ferramentas digitais como experimentação de modelo da cidade para compreensão e legibilidade das dinâmicas urbanas aliadas a diferentes graus do processo participativo. Além disso, busca compreender o contraste entre o planejamento urbano vigente (euclideano, com zonas delimitadas em polígonos que definem usos e padrões de ocupação) e a cidade real, com características nuançadas e que raramente se conformam em polígonos. A partir desta análise, delinea-se uma metodologia de utilização de parâmetros urbanísticos dinâmicos para gerar gradientes com parâmetros continuamente variáveis, estabelecidos na escala do lote. É estudada uma área da cidade de Belo Horizonte para colocar em prática as teorias levantadas e propor formas mais dinâmicas e responsivas de lidar com as cidades.

\section{Zoneamento euclideano e as cidades brasileiras contemporâneas}

A legislação urbanística vigente nas cidades brasileiras baseia-se no Zoneamento Euclideano com a adição de cada vez mais especificidades e números de zonas, que podem ser melhor visualizadas e gerenciadas através de ferramentas digitais que têm se tornado cada vez mais acessíveis e disponíveis para uma melhor compreensão de suas realidades.

O planejamento urbano é tratado até os dias de hoje como um produto acabado, buscando estabelecer ordem, tratando a espacialidade das cidades com mecanismos de intervenção como os de hierarquização e zoneamento, visando controlar a ocupação urbana e os agentes sociais.
No Brasil, durante boa parte do século $X X$, o planejamento sofreu influências do pensamento modernista-racionalista, cujos pressupostos básicos estavam calcados na idéia de modernidade, originária do iluminismo, crença no progresso linear, no discurso universal e o Estado centralizador, capaz de viabilizar usos e funções mais racionais do espaço. Segundo Monte-Mór (2007), "o urbanismo progressistaracionalista propôs autoritariamente um espaço urbano acabado e que visava permitir um rendimento máximo no desempenho das funções urbanas".

A ferramenta de planejamento urbano que predomina nas cidades brasileiras se baseia em delimitar diferentes zonas e prescrever usos e parâmetros de ocupação específicos para cada uma delas. A este processo se dá o nome de Zoneamento Euclideano.

O termo "euclideano" tem origem em uma ação judicial iniciada por uma empresa imobiliária que teve seu terreno zoneado como industrial e processou a cidade de Euclid, em Ohio nos Estados Unidos, em 1922. A Suprema Corte deu ganho de causa à cidade, estabelecendo jurisprudência que permitiria às demais cidades adotar limitações semelhantes no uso e ocupação de seu espaço urbano (Nova York foi a primeira cidade a usar o zoneamento euclideano) e em outros países do mundo (ELLIOT, 2012).

Este processo, segundo Reno (1963), consiste em conceber os zoneamentos como ferramentas reguladoras do uso do espaço urbano, tais como altura dos edifícios, área dos lotes e o redimensionamento do município em regiões que posteriormente seriam os bairros como conhecemos hoje nas cidades brasileiras. 
No inicio de sua existência era comum a utilização de poucos parâmetros, inicialmente três, aplicados homogeneamente a cada zona: o primeiro se definia com os usos, áreas exclusivamente residenciais, comerciais ou industriais. Eram estipuladas hierarquias entre estas áreas: a área residencial era maior que a área comercial, que por sua vez era maior que a industrial; havia, ainda, concessões para que pudessem haver usos mistos em determinadas zonas desde que respeitando tais hierarquias (poderia haver a existência de lotes residenciais em áreas de zoneamento industrial por exemplo, mas jamais o contrário). O segundo parâmetro restringia as alturas máximas de cada área de acordo com as estruturas presentes em cada uma delas. Vale ressaltar que nas regiões periféricas as alturas máximas eram bem inferiores ao permitido nos centros comerciais. O terceiro parâmetro establece as dimensões mínimas de cada lote, com novos loteamentos, mais afastados do centro, tendo áreas mínimas maiores do que as praticadas em zonas residenciais consolidadas em áreas centrais.

Variações destas ferramentas são ainda o núcleo do planejamento urbano nos dias de hoje, com novos parâmetros regulamentadores sendo incorporados na tentativa de responder ao surgimento de novas demandas, como a variação das densidades permitidas de acordo com a capacidade de suporte da infra estrutura urbana.

Assim, com a adição de cada vez mais especificidades na legislação urbanística, diversos questionamentos têm sido apontados na tentativa de propor um processo que se adeque a realidade contemporânea dos meios urbanos.

A busca por novas formas de planejamento urbano aponta para a necessidade de soluções que envolvam os problemas físicos, sociais e ambientais, trazendo consigo um amadurecimento das questões políticas acerca do domínio público.

Segundo John Friedmann (1993), é necessário um grande esforço no âmbito da negociação, mediação e compromisso na tomada de decisões, de forma o novo sistema envolverá, invariavelmente, diferentes formas de participação.

"Above all else, participation requires time. It also requires that both planners and citizens have the capacity to listen sympathetically and share the responsibility for problem definition and solution. The social learning model of planning argues for an open process with two main characteristics: critical feedback and a strong institutional memory. Openness requires democratic procedures. It favors open over closed meetings, and invites criticism and comment." (FRIEDMANN, 1993)

Dessa forma, o estabelecimento de interfaces que fomentem processos participativos é um elemento chave para a construção de espaços urbanos democráticos.

\section{A Cibernética e o Planejamento Urbano}

De acordo com Salomão (2006), a arquitetura funcionalista, mesmo se opondo ao ornamento e à fase estética, retoma uma questão tecnicista mas não Cibernética. Para ela, "o funcionalismo pressupõe uma estrutura fechada e não permite, por exemplo, evoluir, que é uma das características da cibernética". A cidade, o ambiente urbano, está em constante evolução. As cidades crescem, às vezes decrescem, entram em decadência, renascem, etc. O processo contínuo de evolução faz parte do ambiente urbano.

A aproximação entre arquitetura, urbanismo e Cibernética foi feita por Pask (1969). Para Pask "os arquitetos são os primeiros designers de sistema da humanidade". A arquitetura pura busca regular uma complexidade de códigos e traduzí- los em sistemas de comunicação e controle. E o arquiteto nunca precisou se auto denominar um designer de sistemas, já que conceitos de estética e de estilo sempre tiveram lugar na teoria da arquitetura.

"...there are clear limits to what we can control, and great dangers when these limits are exceeded: control is often misapplied so that it takes the form of restriction rather than effective management: and there are advantages in reformulating how we understand the value of control to allow us often to benefit from being out of control." (Glanville, 2002)

Glanville (2002) compara este tipo de controle por restrição a sistemas ditatoriais que são estruturados em relações de poder. Neste contexto o arquiteto opera como ditador da forma que restringe as possibilidades de desenho, apropriação e uso. A ausência de controle pode, assim, ser uma estratégia para expandir o número de opções disponíveis permitindo que sejamos mais criativos.

Para Baltazar (2009) uma alternativa de romper com o controle, restrição e prescrição do desenho é pensar a arquitetura como processo aberto, estabelecendo continuidade entre projeto e uso. Jones (1991) defende este deslocamento do produto acabado para o processo, já que o objetivo do design não deve ser um produto com função determinada, mas sim a própria continuidade do processo de design.

\section{Potenciais das ferramentas digitais aplicadas ao planejamento urbano}

O uso de ferramentas computacionais (como os algoritmos e modelos matemáticos) foi inicialmente introduzido no campo das engenharias para solucionar problemas complexos relacionados a otimização de resultados lógicos.

Para Batty (2011), nas últimas décadas o uso dessas ferramentas tem se tornado crescente no campo da arquitetura, design e mais recentemente, no urbanismo.

O método computacional atualmente não trata apenas da otimização utilitária de resultados, como na otimização de estruturas e orientação de fachadas. Seu principal potencial é permitir ler e simular processos dinâmicos complexos, sendo assim adequado e indispensável para estudos de complexidade das cidades contemporâneas, cujos inúmeros parâmetros de uso podem ser analisados de forma interligada.

"the conception of city has evolved from the machine metaphor of the 20th century to a complex system that is 
analogous to a living organism, exhibiting many of the same characteristics" (WEINSTOCK, 2012)

Embora os métodos computacionais paramétricos sejam frequentemente referenciados apenas por sua dimensão estética - no que é comumente nomeado como "parametricismo, as ferramentas paramétricas permitem sobretudo sistematizar, processar, interpretar e simular elementos da escala urbana e torna-los visíveis em suas diversas dimensões (ambientais, paisagísticas, sociais, econômicas, etc.).

Essa abordagem permite colher e sistematizar dados pra possibilitar ou facilitar a leitura, ao mesmo tempo em que permite fazer simulações de cenários futuros. A dupla função aqui presente (a de fazer leituras complexas e de fazer simulações complexas) é de suma importância para o planejamento das cidades considerando suas dinâmicas cotidianas.

\section{Graus de abertura dos processos que envolvem ferramentas digitais}

As ferramentas paramétricas permitem, por sua natureza, registrar e cruzar uma grande quantidade de dados, ao passo em que a participação/autonomia pede que esses dados sejam legíveis e, portanto, simplificados de alguma forma.

Assim, ainda que a princípio pareça paradoxal aliar os processos participativos a um sistema paramétrico para compreensão do meio urbano, plataformas para a visualização e interação destes dados podem viabilizar uma maior autonomia dos cidadãos na construção da cidade.

Para tanto, é necessária a elaboração de uma metodologia de criação dessa plataforma inicial e a definição dos parâmetros e as formas de abordá-los. Essa plataforma permite agregar mais dados ao mesmo tempo que os torna mais legíveis do que o Zoneamento Euclidiano tradicional permite, viabilizando a visualização, compreensão e atuação no planejamento urbano por diferentes usuários. Viabiliza-se assim que a população desenvolva sua participação na produção do espaço em diferentes graus e em variadas escalas.

A coleta de dados para o uso das ferramentas computacionais pode ocorrer de várias formas diferentes, podendo acontecer por meio de plataformas colaborativas, redes sociais, smartphones, dados públicos dos municípios, etc. Tais ferramentas podem ser utilizadas, portanto, como modos de simplificar determinados sistemas complexos, facilitando sua compreensão e legibilidade; ademais, o próprio fornecimento passivo de dados (como já acontece por meio de aplicativos para smartphones dotados de GPS, por exemplo) já constitui uma forma de participação no processo de planejameto.

Diversas ferramentas e softwares vêm sendo aplicados dentro do processo projetual em diversos escritórios e universidades pelo mundo. Entre os mais utilizados, o presente trabalho buscar explorar as potencialidades e limites do Grasshopper (plugin para Rhinoceros) para o desenvolvimento da metodologia a ser proposta, interligando-o com outras plataformas que sejam pertinentes (GIS, OpenStreetMap, e seus plugins, por exemplo).

Um plugin para Grasshopper com grande potencial para uso no neste tipo de estudo é o "Mosquito", que permite incorporar e cruzar dados de plataformas de dados sociais, financeiras e populares, podendo extrair localização, perfis, imagens e mensagens do Facebook, Twitter, etc.

Este plugin permite ainda a conexão direta e dinâmica com a base cartográfica vetorial do OpenStreetMaps e com dados específicos do Google Maps, incorporando dados e informações conforme as necessidades da plataforma proposta (rotas de carro, bicicletas, distâncias, por exemplo).

Estas ferramentas auxiliam o processo de elaboração dos algoritmos fazendo com que as bases da cidade também possam interagir dinamicamente com o modelo e sobrepor diversas informações da cidade em tempo real, contribuindo para a comparação do existente com as alterações geradas pelos algoritmos, em uma espécie de conexão cibernética entre geometria e dados. Explora as condições espaciais que permitem avaliar o que já aconteceu e o que está acontecendo para criar hipóteses, simular e planejar os desdobramentos urbanos futuros.

\section{Metodologia do metassistema proposto}

A metodologia aqui adotada envolve a pesquisa bibliográfica dos questionamentos levantados e a criação de um metassistema baseado nos parâmetros urbanísticos vigentes na cidade de Belo Horizonte, como modelo inicial, formas de extrapolar tais parâmetros e incorporar novas camadas de informação nesse metassistema. Por fim, analisar como tais ferramentas e suas visualizações simultâneas podem contribuir para formas potencialmente novas de planejamento urbano. 


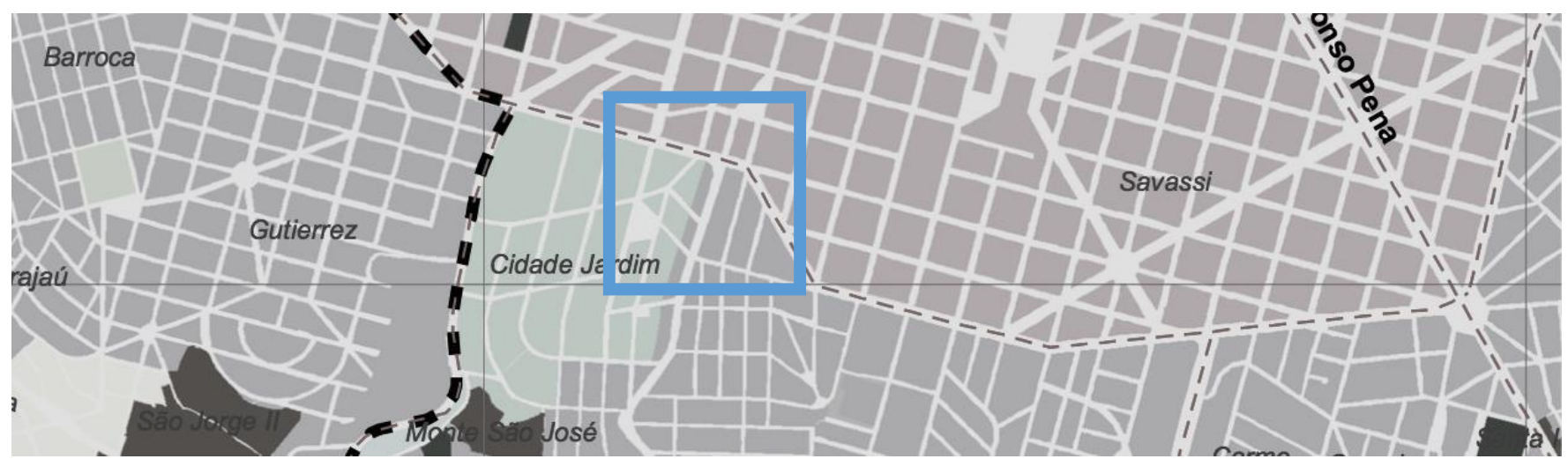

Figura 1: mapa de zoneamento de Belo Horizonte, com a marcação das áreas utilizadas no estudo, entre os bairros Cidade Jardim, Lourdes e Santo Antônio, com as zonas ZP-2, ZCBH e ZA respectivamente.

$\mathrm{Na}$ proposição de algoritimos do metaprojeto tendo Belo Horizonte como estudo de caso para a elaboração do modelo base, optou-se por um trecho da cidade que compreende uma área da região Centro-Sul que tem, em um raio pequeno, grande variedade dos parâmetros urbanísticos definidos pela Lei de Uso e Ocupação do Solo de Belo Horizonte. Foram analisadas 3 quadras que compreendem os Bairros Cidade Jardim, Lourdes e Santo Antônio.

Os parâmetros de ocupação são aqueles que tratam da forma como os edifícios e instalações serão implantados no lote, ou mesmo da restrição à ocupação por construções. Por exemplo, são estabelecidas regras relativas à quantidade de área construída permitida (coeficiente de aproveitamento); à forma como os edifícios podem se espalhar ou se concentrar no lote (taxa de ocupação); a altura que os edifícios devem ter (frequentemente a altura deriva de uma relação com a largura da via); à posição que os edifícios podem ter no lote (recuos frontais, laterais e fundos): à necessidade de garantir qualidade ambiental (taxa de permeabilidade, vegetação, cobreturas verdes, etc): dentre outras regras.

Incorporando as pesquisas teóricas e análises já apresentadas em um único sistema, procedeu-se à criação de um modelo digital de um trecho da cidade de Belo Horizonte com suas características reais para a elaboração de um metassistema.

A partir daí, foram analisados e incorporados ao modelo os parâmetros urbanísticos vigentes no município para melhor compreensão da realidade e delimitação de quais parâmetros poderiam ser extrapolados e quais deveriam ser mantidos.

Como meta, esperou-se que ao longo do processo de análise das quadras e de cada lote individualmente houvesse a alteração dinâmica dos parâmetros envolvidos, em uma reação em cadeia do funcionamento do sistema na medida em que os diferentes parâmetros se influenciam mutuamente.

Iniciando a elaboração dos algoritmos, optou-se pela inserção dos parâmetros iniciais mencionados no capítulo anterior (Coeficiente de Aproveitamento, Altimetria e
Afastamentos) respeitando os índices existentes na legislação atual.

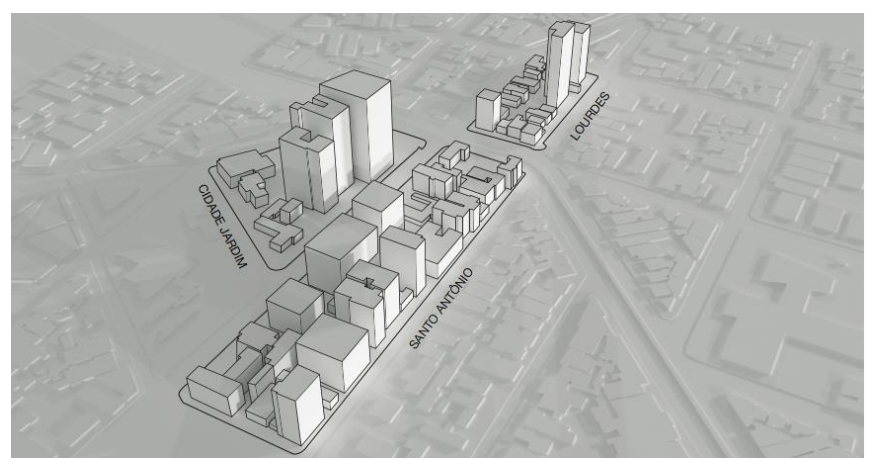

Figura 2: quarteirões para aplicação do metassistema com os parâmetros de coeficiente de aproveitamento, altimetria e afastamentos.

O metassistema permite que estes diferentes parâmetros sejam extrapolados de forma dinâmica. Estabeleceu-se inicialmente, então, um valor único para os três zoneamentos. A partir das peculiaridades de cada trecho, o sistema vai ser reorganizando e se adaptando.

$\mathrm{Na}$ medida em que novos parâmetros passam a ser incorporados no sistema, a complexidade aumenta. A simulação dos resultados possíveis com este aumento de complexidade torna-se exponencialmente mais complexa. No entanto, sua modelagem no metassistema proposto permite que 0 modelo se altere dinamicamente e de forma instantânea na medida em que os parâmetros se modificam.

Utilizando outros parâmetros como elementos desse novo sistema, podemos incorporar como exemplo 0 sombreamento das vias e lotes adjacentes, a exemplo do que é adotado em leis urbanísticas como a da cidade de Denver, nos EUA.

O cálculo do sombreamento dos espaços públicos se relaciona diretamente com a altura e afastamento dos edifícios em seus respectivos lotes, tornando-se uma nova camada de informações a serem incorporadas nos algoritmos do metassistema. 

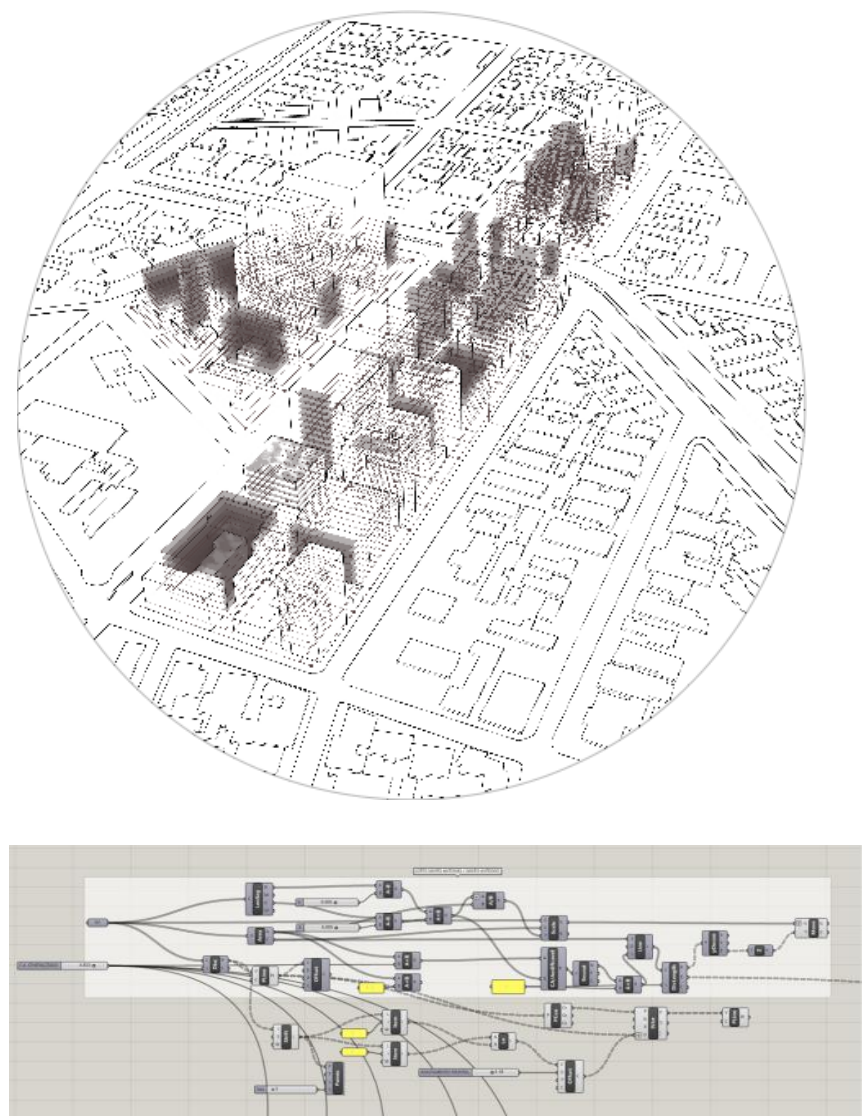

Figuras 3 e 4: metassistema com algoritmos do Grasshopper utilizando os parâmetros iniciais de altimetria, afastamentos e coeficiente de aproveitamento.

Em uma outra relação entre os parâmetros iniciais e dados ambientais como os de sombreamento já citados, pode-se ainda agregar maior complexidade ao modelo utilizando a ferramenta Galapagos, um componente do grasshopper que auxilia na otimização dos algoritmos.

O resultado é um modelo híbrido feito de dados vetoriais e metadados que permite explorar as ferramentas de modelagem paramétrica em associação com uma nova dimensão informativa.

Se, ao longo do processo, o mapeamento vetorial do OpenStreetMap for atualizado, corrigido ou modificado para incorporar alterações produzidas no espaço urbano, o metassistema pode ser atualizado e redesenhar todo 0 processo de forma instantânea.

Assim,o Grasshopper assume o papel de uma plataforma extremamente flexível para organizar em um único fluxo de dados, de grande escala, que vêm de diferentes fontes, descrevendo a sua relação através de regras matemáticas, mas também contando com a avançada capacidade de modelagem 3D oferecida pelo Rhinoceros.

A oportunidade de uma comparação em tempo real de muitos cenários alternativos, obtidos mudando a influência mútua dos parâmetros do sistema, é um método científico para um design mais sensível de nossas cidades, considerando suas reais necessidades e garantindo a coerência com as restrições e metas do projeto.
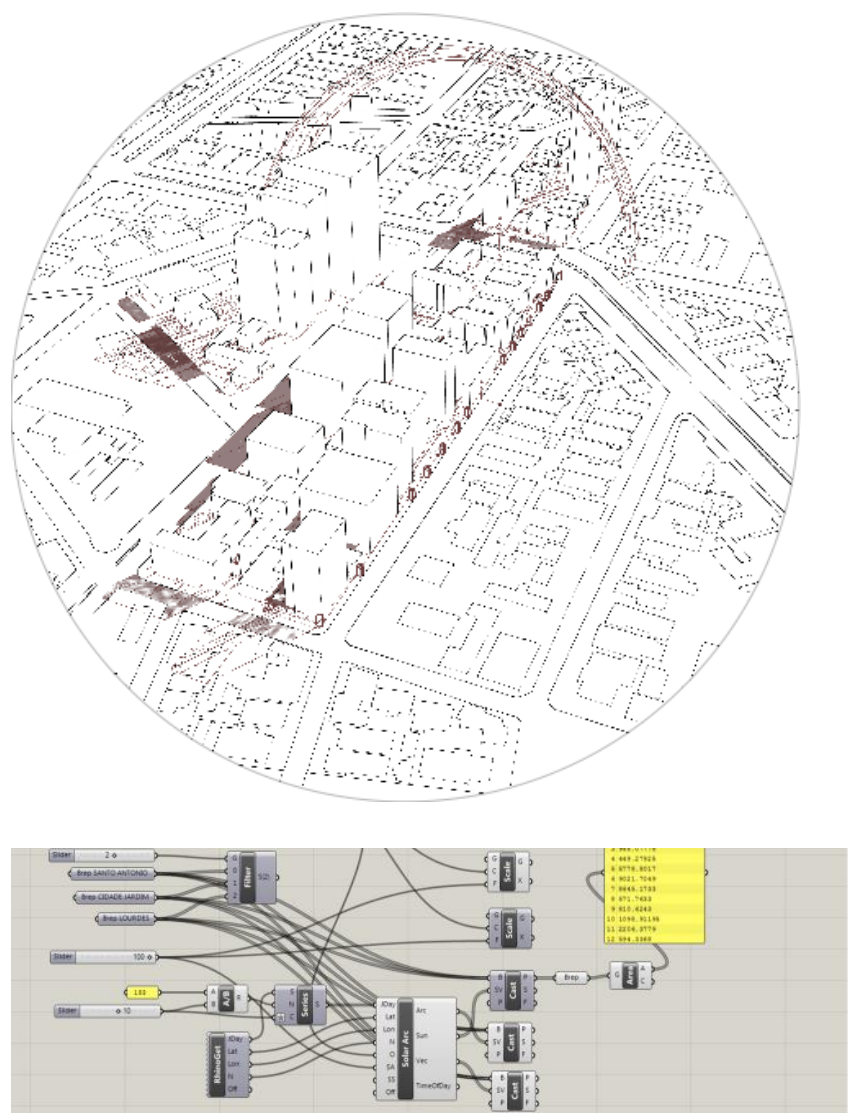

Figura 5 e 6: metassistema com algoritmos do Grasshopper utilizando a sobreposição dos parâmetros iniciais e o de sombreamento.

\section{Conclusão}

Ao longo do processo de compreensão das dinâmicas das cidades, dos graus de participação existentes e dos que podem ser alcançados com a mudança de paradigmas e da forma com que lidamos com o planejamento urbano, ainda que as experimentações propostas sejam simplificadas, elas são aqui apresentadas como uma proposta inicial rumo a uma maior autonomia na construção das cidades.

Este é então um potencial que pode ser explorado na tentativa de questionar o status quo e explorar a utilização das tecnologias e ferramentas digitais vigentes como meio de lidar de uma forma mais simples com sistemas e informações complexas - tanto em sua leitura quanto na atuação sobre eles.

A simples existência das leis não garante a democratização e maior equilíbrio socioambiental para as cidades brasileiras, se estas leis se limitarem a garantir, burocraticamente, acesso a dados sobre os quais a população não tem nem conhecimento e nem autonomia para entender e moldar. Para que isso efetivamente ocorra, é necessário que suas premissas saiam do texto e adentrem nas práticas de planejamento e gestão urbana e, principalmente, sejam incorporadas pelo conjunto da sociedade de forma legível. 
O intuito deste trabalho não é emitir um modelo abrangente e genérico para uma nova abordagem para o planejamento e desenho urbano, mas delimitar os questionamentos pertinentes para que possamos começar a pensar em questões como a regeneração urbana, assentamentos informais e maciça urbanização, participação da comunidade e de representação no espaço, de uma maneira diferente.

Isso não significa, necessariamente, começar do zero, já que as próprias premissas sobre as quais este trabalho se baseia (como os parâmetros urbanísticos) têm lastro na forma de planejamento urbano atualmente vigente. Não se propõe aqui buscar uma nova abordagem, ou fazer tabula rasa como aconteceu ao longo da história ao se propor algo novo; devese, em vez disso, procurar a abordagem adequada para aprimorar a compreensão e o desenvolvimento das cidades com suas dinâmicas e realidades intrínsecas.

Espera-se que os resultados obtidos neste trabalho sejam efetivamente a ponte inicial entre a realidade atual e a inserção das ferramentas digitais em prol de um planejamento urbano mais transparente, aberto e quem sabe no futuro, mais autônomo. Não é possível e nem desejável definir os rumos que tais hipóteses possam tomar, pois seria a limitação dos processos - na contramão do que é proposto.

"So my image of the city remains incomplete, and I think that is proper, because no one should have a final say about the good city. Utopian thinking is an ongoing, time-binding discourse intended to inform our striving. It is no more than that, but also nothing less." (FRIEDMANN, 2000)

\section{Referências}

Batty, M. (2013). The new science of cities. Cambridge, Massachusetts: MIT Press.
Belo Horizonte. Região Metropolitana de Belo Horizonte. Manual Técnico de Edificações, 2012, PBH. Disponível em: http://www.pbh.gov.br/smaru/Manual_Tecnico_Edificacoes/Manu al_Tecnico_Edificacoes_05_09_11.pdf

Elliot, D. L. A Better Way to Zone: Ten Principles to Create More Livable Cities. Island Press, 2012, p. 15-39.

Friedmann, J. Towards a non-euclidean mode of Planning. Journal of the American Planning Association, Vol. 59, No 4, Outono 1993. American Planning Association, Chicago, IL, USA.

Friedmann, J. The Good City: In Defense of Utopian Thinking. International Journal of Urban and Regional Research Volume 24.2 June 2000.

Glanville, R. On being out of control, 2002 (não publicado).

Jones, J. C. (1991). Part 2 Opus one, number two Designing designing, London: Architecture design and technology press (pp. 158-166).

Kapp, S., \& Baltazar, A. P. Por uma Arquitetura não Planejada: o arquiteto como designer de interfaces e o usuário como produtor de espaços. Impulso, Piracicaba, 17(44): 93-103, 2006.

Monte-Mór, R. L. Planejamento urbano no Brasil; emergência e consolidação. Etc... espaço, tempo e crítica, vol. 1, no1, 2007.

Pask, G. The Architectural Relevance of Cybernetics. in: Architectural Design. Londres: Set/1969.

Reno, R. R. Non-Euclidean Zoning: the Use of the Floating Zone, 23 Md. L. Rev. 105 (1963).

Salomão, C. C. Arquitetura e Cibernética. Tese de doutorado - USP, São Paulo. 2006.

Weinstock, M., Gharlrghi, \& M. (July/August 2013) Intelligent Cities and the Taxonomy of Cognitive Scales. Architectural Design. Special Issue: System City: Infrastructure and the Space of Flows Volume 86 - Issue:4, p. 56-65, 